\title{
Epidemiology of nosocomial carbapenem-resistant Klebsiella pneumonia bloodstream infections in south China: factors related to the patient mortality
}

\section{Xiaona Xie}

Wenzhou Medical University First Affiliated Hospital

\section{Xueding Cai}

Wenzhou Medical University First Affiliated Hospital

Tingting Wan

Wenzhou Medical University First Affiliated Hospital

Lianyou Shao

Wenzhou Medical University First Affiliated Hospital

\section{Lijiang Chen}

Wenzhou Medical University First Affiliated Hospital

\section{Dan Yao}

Wenzhou Medical University First Affiliated Hospital

Cheng Ding

Wenzhou Medical University First Affiliated Hospital

\section{Guoping Li}

Tongde Hospital Of Zhejiang Province

\section{Liangxing Wang}

Wenzhou Medical University First Affiliated Hospital

Xiaoying Huang ( $\nabla$ zjwzhxy@126.com )

\section{Research}

Keywords: Carbapenem-resistant Klebsiella pneumoniae, Bloodstream infection, Risk factors, Nosocomial infection, Epidemiology

Posted Date: January 29th, 2020

DOI: https://doi.org/10.21203/rs.2.22227/v1

License: (c) (i) This work is licensed under a Creative Commons Attribution 4.0 International License.

Read Full License 


\section{Abstract}

Background: Carbapenem-resistant Klebsiella pneumoniae (CRKP) causing Bloodstream infection (BSI) are associated with high rates of mortality . Nevertheless, only a few studies regarding the epidemiology of CRKP BSI in south China. The purpose of this study was to describe the epidemiology, clinical characteristics, and the mortality of risk factors associated with CRKP causing bloodstream infection.

Methods: A retrospective study of patients with CRKP BSI was recruited from teaching hospital in south China from January 2016 to December 2018. Clinical data were collected from medical records.

Results: In total, 90 patients with CRKP BSI were enrolled in the study, while $57 \%$ (51/90) of the CRKP BSI were obtained from ICU. Most CRKP BSIs originated from hospitals $(81 ; 85 \%)$, while the rest $(9 ; 10 \%)$ were healthcare-associated . In univariate analysis, gastrointestinal hemorrhage $(p=0.029)$, Pitt bacteremia score $(P=0.045)$, Charlson comorbidity index $(p=0.018)$ and Corticosteroids use $(p=0.036)$ and Septic shock $(p=0.001)$ were associated with the risk factors for mortality. In a multivariate analysis, septic shock (adjusted odds ratio [aOR] 5.591, 95\% confidence interval [CI] 1.405-22.246, $\mathrm{P}=0.015$ ) and Corticosteroids use (aOR 4.148, 95\% Cl 1.331-12.928, $\mathrm{P}=0.014)$ were independently predictors of mortality.

Conclusion: Our data showed that the morbidity and mortality of CRKP BSIs patient from ICU and non-ICU was no significant difference. Standardizing operation and improving nurse quality may play an important role in CRKP BSI patient in intensive care unit. Septic shock and Corticosteroids use were the independent factors of CRKP BSI patient mortality. However, the study did not show an association between invasive procedures and the development of CRKP BSI.

\section{Introduction}

Klebsiella pneumoniae isolates are among the most common bacteria causing hospital acquired infections, including bloodstream infections (BSIs) [1, 2]. Carbapenem-resistant K. pneumoniae (CRKP) was originally identified in 1996 and quickly became a serious health care problem in many countries, including China[3, 4]. CRKP can cause many serious infections, including, soft tissue infections, pneumonia, urinary tract infections, intra-abdominal and bloodstream infections. Bloodstream infection (BSI) with carbapenem-resistant Klebsiella pneumoniae (CRKP) is related to particularly high mortality rates[5]. The mortality rate of carbapenem-resistant Klebsiella pneumoniae (CRKP) infections were reported to be $50.06 \%, 46.71 \%, 33.24 \%$, and $44.82 \%$ in Europe, South America, North America, and Asia respectively [6]. Indeed, all-cause mortality from critically ill patients with severe bloodstream CRKP infections is nearly $70 \%[7-9]$. Emerging resistance to antibiotics of last resort (i.e. tigecycline or colistin) leaving very few therapeutic options have been shown in recent studies [10,11]. Increasing antimicrobial drug resistance, especially in carbapenem-resistant Klebsiella pneumonia (CRKP), can limit the choice of antibiotics used for the treatment of infectious diseases and further poses a negative impact on patient 
outcome. The high occurrence of cases and mortality, economic burden and dearth of alternative drugs makes CRKP infections an important threat to public health worldwide.

There are several risk factors associated with the development of CRKP BSI. Risk factors related to CRKP BSI include comorbidities, ICU stay, use of invasive devices, and exposure to carbapenem prior to culture[12]. Due to the extensive use of invasive surgery and the frequent use of antimicrobial agents, ICUs are described as a factory for generate, spread, and expansion of antimicrobial resistance. While regional and institutional infection control programs remain the cornerstone for reducing the risk of patient acquisition and death from MDR pathogens, additional tools are needed to facilitate patient screening, early diagnosis and timely use of appropriate antibiotic treatment regimens. The physician may consider this risk assessment when deciding whether a particular patient should receive early appropriate therapy for CRKP BSI until culture and sensitivity results are available.

To the best of our knowledge, few studies have assessed the hazard factors for mortality of CRKP bloodstream infection (BSI) in south China. In this study, we sought to evaluate the epidemiological, clinical characteristics, the risk factors for mortality of CRKP BSIs in patients admitted to a teaching hospital in Wenzhou, China.

\section{Patients And Methods}

\section{Study design and patients}

From January 2016 to December 2018, we retrospectively analyzed clinical data in the First Affiliated Hospital of Wenzhou Medical University, a tertiary university hospital with 4,100 beds in Zhejiang, southern China. We included all adult patients (age of day $\geq 18$ years) hospitalized in the First Affiliated Hospital of Wenzhou Medical University in south China. BSI onset was defined as the collection date of a positive blood culture. Only the first CRKP BSI was included, and patients with polymicrobial BSI were excluded. All criteria are graded within 48 hours before or on the day of first positive blood culture. Laboratory examination included lymphocytes $(10 * 9 / L)$. The study was approved by the Ethics Committee of the First Affiliated Hospital of Wenzhou Medical University. Because the retrospective study did not cause harm to the patient, informed consent was waived.

\section{Data collection and definitions}

The data of the clinical microbiology laboratory collected included information regarding demographic characteristics, comorbidities, intravascular catheter use, recent (30 days) surgical procedures, the use of Corticosteroids (use Corticosteroids during bloodstream infections), carbapenem exposure (exposure to carbapenems during the 30 days preceding admission to the ICU) and patient outcomes were also collected(Table1). BSIs patients were further classified into death group $(n=60)$ and survivor group $(n=30)$ according to clinical outcome of antimicrobial treatment. BSIs were defined as hospital-acquired if the positive blood culture results more than $48 \mathrm{~h}$ after the admission of patient to the hospital and no symptoms of infection had been noted at admission. If blood culture samples had been collected within 
48h after hospital admission, the isolate was defined as Community-acquired or healthcare-associated [13]. Septic shock was defined as sepsis associated with organ dysfunction and persistent hypotension despite volume replacement[14]. Comorbidities were calculated according to the Charlson's score[15], and the Pitt bacteremia score (PBS) was used to assess disease severity at the time of positive blood culture [16].

\section{Microbiological methods}

We processed bacterial cultures in the clinical microbiology laboratory. Initially, Vitek2 system (BioMèrieux, France) was used to determine bacterial identification and the antimicrobial susceptibility. Then, the isolates were stored in frozen condition at $-80 \circ \mathrm{C}$ with $30 \%$ glycerol. The minimum inhibitory concentration (MIC) values for tested antimicrobial agents were determined by an automated broth dilution method and interpreted by the recommendation of the European Committee on Antimicrobial Susceptibility Testing (EUCAST, 2015).

\section{Statistical analysis}

Statistical analysis was carried out by SPSS for Windows, version 21.0 (IBM, Armonk, NY, USA).

The mean and standard deviation (SD), median and interquartile range (IQR) were used to compare the continuous variables of normally and non-normally distributed, respectively. Students't test (normal distribution) and Mann-Whitney U-test (non-normal distribution) were used to analyze and compare continuous variables. Categorical variables are represented by numbers and percentages. The categorical variables were analyzed by Fisher's exact test. Logistic regression analysis was used to determine the risk factors of death in each variable. In univariate analysis, the significant variables with $P<0.05$ were included in multivariate analysis, in which we evaluated the risk factors for CRKP BSI-related mortality. A $p$ value $<0.05$ was considered to statistically significant.

\section{Results}

\section{Characteristics of patients}

Overall, 90 patients with CRKP BSI met the inclusion criteria. Ultimately, according to the inclusion and exclusion the unique episodes were included in the analysis (Fig 1). The demographic and clinical characteristics of patients with CRKP-BSIs are presented in Table 1. CRKP-BSI cases mainly occurred in subjects aged over 60 years (72\%). The median patient age was 68 years (interquartile range $21-93$ years), and 64 of 90 patients (67\%) were males. All episodes of CPKP BSIs were either hospital acquired $(85 \%)$ or healthcare associated $(10 \%)$, while none was community acquired.

The most common disease was diabetes (27\%), Previous Surgery (44\%) or intracranial disease (30\%), gastrointestinal hemorrhage (20\%), chronic kidney disease (17\%), cardiovascular disease (4\%) and solid tumors $(15 \%)$. There were $3(3 \%)$ cases involving solid organ transplantation and $12(13 \%)$ receiving chemotherapy. Overall, 39\% of patients underwent Corticosteroids use. $12(13 \%)$ patients received 
immunosuppressive therapy (cyclosporine or leflunomide) to treat systemic lupus erythematosus (Fig 2). The median Charlton comorbidity index $(\mathrm{CCl})$ and Pitt bacteremia score at onset were 4 (interquartile range3-5) and 3 (interquartile range 2.0-6.0), respectively. The invasive Procedures was indwelled Urinary catheter $(69 \%)$, indwelled central venous catheter $(72 \%)$, mechanically ventilated $(45 \%)$, gastrointestinal decompression (63\%), renal replacement therapy (14\%) (Fig 2).

\section{Increased rates of CRKP-BSIs, including ICU and Non-ICU patients}

In our study, 90 unique episodes of CRKP-BSI were included, $57 \%$ (51/90) of the CRKP isolates were came from ICU and 43\% (39/90) of the CRKP BSI patients were came from non-ICU. We discovered that the occurrence of bloodstream infection causing nosocomial carbapenem-resistant Klebsiella pneumonia is increasing since 2016, and the rates of infections are much higher than the national average in the hospital included in this study. The percent of CRKP-BSI mortality in this study are presented in Fig 3. The percentage of overall mortality increased from $60 \%$ in 2016 to $65 \%$ in 2017, with the highest rate obtained in 2018, up to $69 \%$ of CRKP BSI patients in our hospital. A similar trend, the rates of CRKP BSI mortality acquired from our Hospital in intensive care unit, rising from 60\% in 2016 to $66.7 \%$ in 2017 and $78.9 \%$ in 2018. Simultaneously, the rates of CRKP BSI mortality acquired from our Hospital in non-ICU, rising from $61.5 \%$ in 2016 to $62.5 \%$ in 2017 , descending $58.8 \%$ in 2018 (Fig 3).

\section{Risk factors for mortality of CRKP BSI patients}

Overall in-hospital crude mortality was 65\% (59/90), similar to the previous studies[17]. Our results indicate that the invasive Procedures was indwelled Urinary catheter (69\%), indwelled central venous catheter $(72 \%)$, mechanically ventilated $(45 \%)$, gastrointestinal decompression $(63 \%)$, renal replacement therapy (14\%) (Table 1). Previous studies have evaluated several risk factors for the mortality associated with CRKP BSI, including use of indwelled Urinary catheter, indwelled central venous catheter, mechanically ventilated and exposure to carbapenems. However, in our analysis, gastrointestinal hemorrhage $(p=0.029)$, Pitt bacteremia score $(P=0.045)$, Charlton comorbidity index $(p=0.018)$ and Corticosteroids therapy $(p=0.036)$ and Septic shock $(p=0.001)$ were associated with risk factors for mortality (Fig 4). In univariate analyses, variables significant at $p<0.05$ were included in the multivariate analysis. On multivariate analyses, septic shock (adjusted odds ratio [aOR] 5.591, 95\% confidence interval $[\mathrm{Cl}]$ 1.405-22.246, $\mathrm{P}=0.015$ ) and Corticosteroids therapy (aOR 4.148, 95\% $\mathrm{Cl} 1.331-12.928, \mathrm{P}=0.014$ ) were independently associated with a risk effect on mortality (Table 2).

\section{Therapeutic characteristics}

As defined in Materials and methods, 53 patients (58.8\%) received early appropriate therapy, and 37 patients who received inappropriate therapy. Fifty-there patients received at least one active antibiotic, including 45 patients who received Tigecycline-based therapy $(84.7 \%, 45 / 53)$, one patient who received Polymyxin B -based therapy and 7 who received Tigecycline combine Polymyxin B $(13.2 \%, 7 / 53)$. The clinical characteristics of patients who underwent Tigecycline-based therapy, Polymyxin B -based therapy and Tigecycline combine Polymyxin B were summarized in FIG 4. 


\section{Discussion}

In recent years, carbapenem-resistant Klebsiella pneumoniae (CRKP) associated bacterial infections have spread worldwide becoming a serious global public health problem in a number of countries, including China. Surveillance of antibiotic resistance by the China CHINET reported that the rate of carbapenemresistant Klebsiella pneumoniae isolates increased from $2.4 \%$ in 2005 to $13.4 \%$ in 2014[18]. The studies were to demonstrate the epidemiological, clinical characteristics and the risk factors for predictors of mortality in south china. While our study has been collected in a single medical center, the samples were date from the First Affiliated Hospital of Wenzhou Medical University, which is one of the largest integrated teaching hospitals, clinical practice and scientific research.

CRKP-BSI cases mainly occurred in aged over 60 years $(72 \%)$ and more frequently in males $(67 \%)$ than in females. Confirmation of this finding is also available in the European Centers for Disease Control and Prevention database, where the higher proportion of carbapenem-resistant K. pneumoniae in males is particularly evident in countries at high prevalence of carbapenem resistance such as Italy and Romania. The reason for the higher incidence of CRKP-BSI in males than in females is not clear, but it is worth noting that the higher incidence of sepsis in males is associated with genetic susceptibility [19]. In our study, we observed an increase in CRKP-BSI with age. This increase, especially for patients 60 years of age and older, may be associated with the high vulnerability in the elderly[20], which may determine higher frequencies and longer hospital stays than younger patients.

The patient population affected by CPKP BSI had several evident characteristics; all episodes of BSIs were either hospital or health care associated. The data showed that the rate of hospital-acquired infections was $66 \%$, however, $75 \%$ of the patients who died had hospital-acquired infection from the CRE Epicenter of the United States[21]. Our data showed that the rate of $90 \%$ CRKP BSI patients had hospitalacquired infection, while none was community acquired. It is important for physician to control hospital infection as soon as possible.

Carbapenem-resistant Klebsiella pneumoniae (CRKP) Bloodstream infections(BSI) are becoming an crisis of global dimensions, including ICU and Non-ICU patients, due to high morbidity and mortality[22]. In our study, $57 \%$ (51/90) of the CRKP isolates were came from ICU and $43 \%$ (39/90) of the CRKP BSI patients were came from non-ICU. The overall mortality obviously increased from $60 \%$ in 2016 to $65 \%$ in 2017 , with the highest rate obtained in 2018 , up to $69 \%$. This discrepancy probably caused by Zhejiang Province is one of the highest CRKP infections prevalence's region in China[18]. It is a major clinical finding that the morbidity and mortality of CRKP BSIs patient in ICU and non-ICU was no significant difference. A possible explanation may be that standardizing operation and improving nurse quality is vital key to the critically CRKP BSI patient in intensive care unit. Furthermore, characterized by advanced technology to care for severely patients and early appropriate therapy in intensive care unit, which may lead to the difference from previous data[23]. It is a monocentric study that our data may be different from other centers. Therefore, further multi-centric studies are needed to confirm our findings. 
Bloodstream infections causing by Carbapenem-resistant Klebsiella pneumoniae (CRKP) clinical characteristics have been attributed to the various aspects. In previous study, it is more frequent among patients associated with CRKP BSIs with severe chronic comorbidities, but chronic comorbidities were not risk factors for CRKP BSI[24]. As consistently shown in our studies, comorbidities were not independent risk factors for CRKP BSI. Furthermore, our studies show that indwelling central venous catheter and urinary catheter were not independent risk factors for CRKP BSI, which is different with previous studies. The reason for this might associated with the small number of CRKP BSI cases in the study. All the patient peripheral blood lymphocytes before bloodstream infection were decreased in CRKP in this study. It may help physicians pay more attention to the patients who have a higher risk of bloodstream infection when the lymphocytes decreased.

The mortality rates associated with CRKP BSI have been widely reported and they range from $40 \%$ to $70 \%$ $[25,26]$. Overall, the rate of mortality in our patients with CRKP BSI was higher $(65 \%)$ compared to the findings of previously studies [27]. Previous studies have evaluated several risk factors for the mortality associated with CRKP BSI, including use of corticosteroid, use of invasive devices, septic shock and exposure to carbapenems[27-29]. Fig-4 shows that CRKP BSI was related to gastrointestinal hemorrhage, Pitt bacteremia score, Charlton comorbidity index and Corticosteroids use and Septic shock. Septic shock and Corticosteroids use were independent risk factors for CRKP BSI, which is different with previous reported. The reason for this might provide an explanation that the regional differences and effective Hospital Infection-Control of CRKP BSI in our study. Corticosteroid should be added to septic shock patients according to the Surviving Sepsis Campaign guidelines[30]. In our study, an alarming finding is that the use of corticosteroids as adjunctive therapy in CRKP infections has deleterious effects. The most important risk factor for mortality in our study was septic shock at CRKP BSI onset; not surprisingly, this variable had been found to be associated with mortality in several previous studies performed on general population patients with CRKP BSI[8, 31].

There were several limitations associated with this study. First, the current study was a retrospective, single-center study including 90 patients with clinical analysis. Second, Clinical data were obtained retrospectively from medical records, and there may be some differences in physician practices or accuracy of information. Finally, prospective and further multi-centric studies are needed to confirm our findings.

\section{Conclusion}

There was no significant difference in CRKP BSIs patient morbidity and mortality between ICU and nonICU. Standardizing operation and improving nurse quality may play an important role in CRKP BSI patient in intensive care unit. Septic shock and Corticosteroids therapy were independent risk factor related to the patient mortality. However, the present study did not show an association between invasive procedures and the development of CRKP BSI, which is different with previous studies reported. The regional differences and effective Hospital Infection-Control of CRKP BSI in our study might be the reason. 


\section{Declarations}

\section{Acknowledgment}

For providing CRKP isolates and reviewing medical records, we thank

\section{Author contributions}

All authors contributed to data analysis, drafting and revising the article, gave final approval of the version to be published, and agree to be accountable for all aspects of the work.

\section{Availability of data and materials}

All data generated or analyzed during this study are included in this

published article.

\section{Funding}

Not applicable.

\section{Consent for publication}

Not applicable.

\section{Competing interests}

The authors declare that they have no competing interests.

\section{Ethics approval and consent to participate}

This study was approved by the First Affiliated Hospital of Wenzhou Medical University Ethics Committee. Informed consent was not needed due to the retrospective nature of the study; additionally, the patient data accessed inthis research was anonymous. Therefore, the First Affiliated Hospital of Wenzhou Medical University Ethics Committee waived the need for consent.

\section{References}

1. LS M-P, L P, RA B, MJ S, GL D, M C, G C, J G, M G, MK H et al: Clinical epidemiology of the global expansion of Klebsiella pneumoniae carbapenemases. The Lancet Infectious diseases 2013, 13(9):785-796.

2. $P$ N, G C, T N: The real threat of Klebsiella pneumoniae carbapenemase-producing bacteria. The Lancet Infectious diseases 2009, 9(4):228-236.

3. Giannella M, Bartoletti M, Morelli MC, Tedeschi S, Cristini F, Tumietto F, Pasqualini E, Danese I, Campoli C, Lauria ND et al: Risk factors for infection with carbapenem-resistant Klebsiella 
pneumoniae after liver transplantation: the importance of pre- and posttransplant colonization. $A m \mathrm{~J}$ Transplant 2015, 15(6):1708-1715.

4. MacKenzie FM, Forbes KJ, Dorai-John T, Amyes SGB, Gould IM: Emergence of a carbapenemresistant Kiebsiella pneumoniae. The Lancet 1997, 350(9080).

5. Pierluigi Viale, Maddalena Giannella, Russell Lewis, Emanuele Maria, Trecarichi, Nicola Petrosillo, Tumbarello M: Predictors of mortality in multidrug-resistant Klebsiella pneumoniae bloodstream infections. Expert Rev Anti Infect Ther 2013, 11(10):1053-1063.

6. Xu L, Sun X, Ma X: Systematic review and meta-analysis of mortality of patients infected with carbapenem-resistant Klebsiella pneumoniae. Ann Clin Microbiol Antimicrob 2017, 16(1):18.

7. Borer A, Saidel-Odes L, Riesenberg K, Eskira S, Peled N, Nativ R, Schlaeffer F, Sherf M: Attributable mortality rate for carbapenem-resistant Klebsiella pneumoniae bacteremia. Infect Control Hosp Epidemiol 2009, 30(10):972-976.

8. Daikos GL, Tsaousi S, Tzouvelekis LS, Anyfantis I, Psichogiou M, Argyropoulou A, Stefanou I, Sypsa V, Miriagou V, Nepka M et al: Carbapenemase-producing Klebsiella pneumoniae bloodstream infections: lowering mortality by antibiotic combination schemes and the role of carbapenems. Antimicrob Agents Chemother 2014, 58(4):2322-2328.

9. Falagas ME, Tansarli GS, Karageorgopoulos DE, Vardakas KZ: Deaths attributable to carbapenemresistant Enterobacteriaceae infections. Emerg Infect Dis 2014, 20(7):1170-1175.

10. van Duin D, Cober ED, Richter SS, Perez F, Cline M, Kaye KS, Kalayjian RC, Salata RA, Evans SR, Fowler VG, Jr. et al: Tigecycline therapy for carbapenem-resistant Klebsiella pneumoniae (CRKP) bacteriuria leads to tigecycline resistance. Clin Microbiol Infect 2014, 20(12):01117-1120.

11. van Duin D, Doi Y: Outbreak of Colistin-Resistant, Carbapenemase-Producing Klebsiella pneumoniae: Are We at the End of the Road? J Clin Microbio/ 2015, 53(10):3116-3117.

12. van Duijn PJ, Verbrugghe W, Jorens PG, Spöhr F, Schedler D, Deja M, Rothbart A, Annane D, Lawrence C, Nguyen Van J-C et al: The effects of antibiotic cycling and mixing on antibiotic resistance in intensive care units: a cluster-randomised crossover trial. The Lancet Infectious Diseases 2018, 18(4):401-409.

13. Hussein K, Raz-Pasteur A, Finkelstein R, Neuberger A, Shachor-Meyouhas Y, Oren I, Kassis I: Impact of carbapenem resistance on the outcome of patients' hospital-acquired bacteraemia caused by Klebsiella pneumoniae. J Hosp Infect 2013, 83(4):307-313.

14. RP D, MM L, A R, D A, H G, SM O, JE S, CL S, IS D, R J et al: Surviving Sepsis Campaign: international guidelines for management of severe sepsis and septic shock, 2012. Intensive care medicine 2013, 39(2):165-228.

15. Charlson ME, Pompei P, Ales KL, MacKenzie CR: A new method of classifying prognostic comorbidity in longitudinal studies: development and validation. J Chronic Dis 1987, 40(5):373-383.

16. Korvick JA, Bryan CS, Farber B, Beam TR, Schenfeld L, Muder RR, Weinbaum D, Lumish R, Gerding DN, Wagener MM: Prospective Observational Study of Kiebsiella Bacteremia in 230 Patients: 
Outcome for Antibiotic Combinations versus Monotherapy. Antimicrob Agents Chemother 1992, 36(12):2639-2644.

17. MJ S, S K-L, S N-V, D S, A L, Y C: Predictors of carbapenem-resistant Klebsiella pneumoniae acquisition among hospitalized adults and effect of acquisition on mortality. Antimicrobial agents and chemotherapy 2008, 52(3):1028-1033.

18. FP H, Y G, DM Z, F W, XF J, YC X, XJ Z, CX Z, P J, Y X et al: Resistance trends among clinical isolates in China reported from CHINET surveillance of bacterial resistance, 2005-2014. Clinical microbiology and infection : the official publication of the European Society of Clinical Microbiology and Infectious Diseases 2016, null(undefined):S9-14.

19. JA H, F S, D F, M B, S W, M R, G R, G S: Gene variants of the bactericidal/permeability increasing protein and lipopolysaccharide binding protein in sepsis patients: gender-specific genetic predisposition to sepsis. Critical care medicine 2001, 29(3):557-561.

20. NP W: Aging of the immune system: how much can the adaptive immune system adapt? Immunity 2006, 24(5):495-499.

21. MJ S, L C, G P, A G-S, G W, AC K, SK S, ME R, SJ S, SG J et al: Multicenter Clinical and Molecular Epidemiological Analysis of Bacteremia Due to Carbapenem-Resistant Enterobacteriaceae (CRE) in the CRE Epicenter of the United States. Antimicrobial agents and chemotherapy 2017, 61(4): undefined.

22. Alicino C, Giacobbe DR, Orsi A, Tassinari F, Trucchi C, Sarteschi G, Copello F, Bono VD, Icardi CVaG: Trends in the annual incidence of carbapenem-resistant Klebsiella pneumoniae bloodstream infections: a 8-year retrospective study in a large teaching hospital in northern Italy: BMC Infectious Diseases 2015.

23. AF S: Review of studies of the impact on Gram-negative bacterial resistance on outcomes in the intensive care unit. Critical care medicine 2009, 37(4):1463-1469.

24. K H, A R-P, R F, A N, Y S-M, I O, I K: Impact of carbapenem resistance on the outcome of patients' hospital-acquired bacteraemia caused by Klebsiella pneumoniae. The Journal of hospital infection 2013, 83(4):307-313.

25. D. Ben-David, R. Kordevani, Keller N, I. Tal, Marzel A: Outcome of carbapenem resistant Klebsiella pneumoniae bloodstream infections. Clin Microbiol Infect 2012, 18(1).

26. Patel G, Huprikar S, Factor SH, Jenkins SG, Calfee DP: Outcomes of carbapenem-resistant Klebsiella pneumoniae infection and the impact of antimicrobial and adjunctive therapies. Infect Control Hosp Epidemiol 2008, 29(12):1099-1106.

27. Tian L, Tan R, Chen Y, Sun J, Liu J, Qu H, Wang X: Epidemiology of Klebsiella pneumoniae bloodstream infections in a teaching hospital: factors related to the carbapenem resistance and patient mortality. Antimicrob Resist Infect Control 2016, 5:48.

28. Papadimitriou-Olivgeris M, Fligou F, Bartzavali C, Zotou A, Spyropoulou A, Koutsileou K, Vamvakopoulou S, Sioulas N, Karamouzos V, Anastassiou ED et al: Carbapenemase-producing 
Klebsiella pneumoniae bloodstream infection in critically ill patients: risk factors and predictors of mortality. Eur J Clin Microbiol Infect Dis 2017, 36(7):1125-1131.

29. Papadimitriou-Olivgeris M, Marangos M, Fligou F, Christofidou M, Sklavou C, Vamvakopoulou S, Anastassiou ED, Filos KS: KPC-producing Klebsiella pneumoniae enteric colonization acquired during intensive care unit stay: the significance of risk factors for its development and its impact on mortality. Diagn Microbiol Infect Dis 2013, 77(2):169-173.

30. RP D, MM L, A R, D A, H G, SM O, JE S, CL S, IS D, R J et al: Surviving sepsis campaign: international guidelines for management of severe sepsis and septic shock: 2012. Critical care medicine 2013, 41(2):580-637.

31. Tumbarello M, Viale P, Viscoli C, Trecarichi EM, Tumietto F, Marchese A, Spanu T, Ambretti S, Ginocchio F, Cristini F et al: Predictors of mortality in bloodstream infections caused by Klebsiella pneumoniae carbapenemase-producing K. pneumoniae: importance of combination therapy. Clin Infect Dis 2012, 55(7):943-950.

\section{Tables}

Table 1

\begin{tabular}{|c|c|c|c|c|}
\hline Characteristics & $\begin{array}{l}\text { All patients } \\
(\mathrm{N}=90)\end{array}$ & $\begin{array}{l}\text { Survivor group } \\
(\mathrm{n}=30)\end{array}$ & $\begin{array}{l}\text { Death group } \\
(\mathrm{n}=60)\end{array}$ & P-value \\
\hline Age (years), median(IOR) & $68(58-74)$ & $63(55-69)$ & $70(61-75)$ & 0.599 \\
\hline Hospital-acquired & 81(85) & 27(90) & $54(83)$ & 0.963 \\
\hline Healthcare-associated & $9(10)$ & $3(10)$ & $6(9)$ & 0.342 \\
\hline Lymphocyte & $.56(0.22-0.85)$ & $0.68(0.39-0.90)$ & $0.51(0.21-0.82)$ & 0.702 \\
\hline Intracranial disease & $27(30)$ & $10(32)$ & 17(29) & 0.950 \\
\hline Cardiovascular disease & $4(4)$ & $2(6)$ & $2(3)$ & 0.442 \\
\hline Diabetes mellitus & $24(27)$ & $8(26)$ & $16(27)$ & 0.836 \\
\hline Previous Surgery ${ }^{a}$ & $40(44)$ & $17(55)$ & $23(39)$ & 0.063 \\
\hline Gastrointestinal hemorrhage & $19(20)$ & $2(7)$ & $17(29)$ & 0.003 \\
\hline Immunosuppressive therapy ${ }^{\mathrm{C}}$ & $12(13)$ & $5(16)$ & $7(12)$ & 0.608 \\
\hline CCI, median (IQR) & $4(3-5)$ & $3(2-5)$ & $4(3-6)$ & 0.172 \\
\hline \multicolumn{5}{|l|}{ Invasive Procedures } \\
\hline Indwelled Urinary catheter & $69(77)$ & $22(71)$ & $47(80)$ & 0.753 \\
\hline Indwelled central venous cathete & $72(80)$ & $22(71)$ & $50(85)$ & 0.328 \\
\hline Mechanically ventilated & $45(50)$ & $15(48)$ & $30(51)$ & 0.876 \\
\hline Surgical drainage & 27(19) & $5(16)$ & $12(20)$ & 0.568 \\
\hline Renal replacement therapy & 14(16) & $4(13)$ & $10(17)$ & 0.673 \\
\hline Gstrointestinal decompression & $63(70)$ & $20(65)$ & $43(73)$ & 0.741 \\
\hline \multicolumn{5}{|l|}{ Severity of Illness } \\
\hline Septic shock & $4(38)$ & $3(10)$ & $31(53)$ & 0.000 \\
\hline PBS, median(IQR) & $3(2-6)$ & $2(1-4)$ & $3(2-8)$ & 0.128 \\
\hline Corticosteroids use & $35(39)$ & $9(29)$ & $26(44)$ & 0.003 \\
\hline Chemotherapy, radiotherapy & $12(13)$ & $4(13)$ & $8(14)$ & 0.102 \\
\hline Length of hospitalization & $36(19-56)$ & $52(36-64)$ & $27(16-48)$ & 0.047 \\
\hline
\end{tabular}


Table 2

\begin{tabular}{lcccc}
\hline \multirow{2}{*}{ Variable } & \multicolumn{2}{c}{ Univariate analysis } & \multicolumn{2}{c}{ Multivariate analysis } \\
\cline { 2 - 5 } & Odds ratio(OR)(95\%CI) & P-value & Odds ratio(OR)(95\%CI) & P-value \\
\hline PBS & $1.588(1.003-1.394)$ & 0.045 & $1.071(0849-1.350)$ & 0.563 \\
CCI & $1.314(1.047-1.650)$ & 0.018 & $1.248(0.943-1.651)$ & 0.122 \\
Gastrointestinal hemorrhage & $5.535(1.186-25.832)$ & 0.029 & $3.763(0.672-21.058)$ & 0.131 \\
Septic shock & $9.621(2.633-35.154)$ & 0.001 & $5.591(1.405-22.246)$ & 0.015 \\
Corticosteroids use & $2.875(1.072-7.710)$ & 0.036 & $4.148(1.331-12.928)$ & 0.014 \\
\hline
\end{tabular}

\section{Figures}

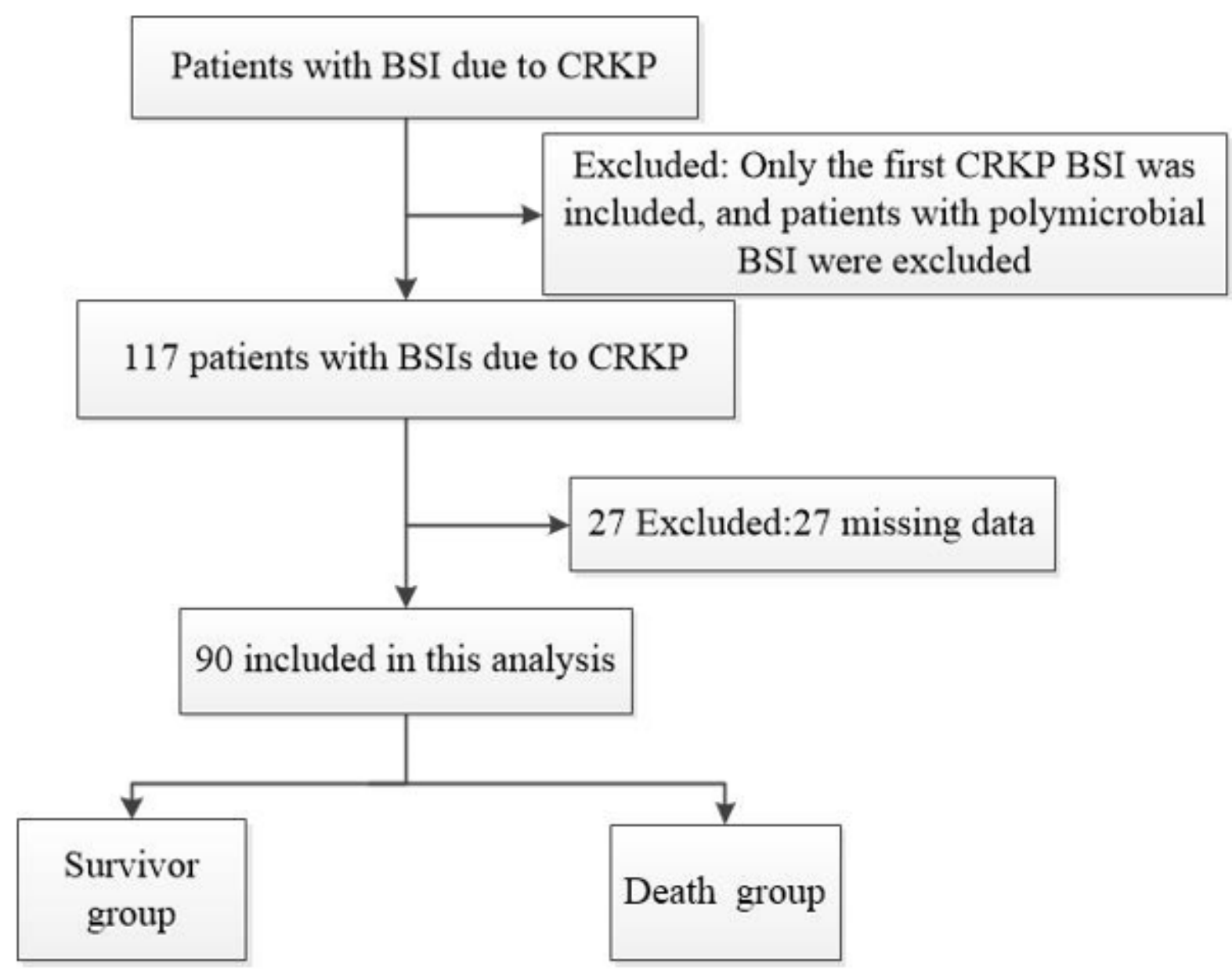

Figure 1

Flowchart of the included patients with BSIs due to CRKP 


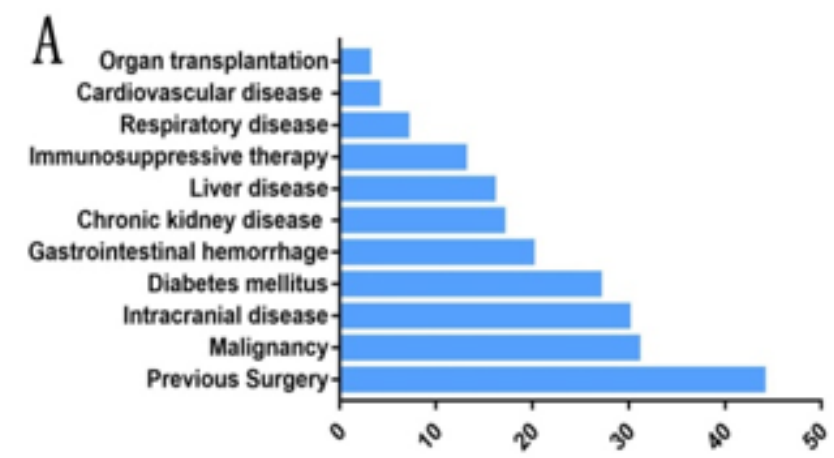

percentage of Comorbidities

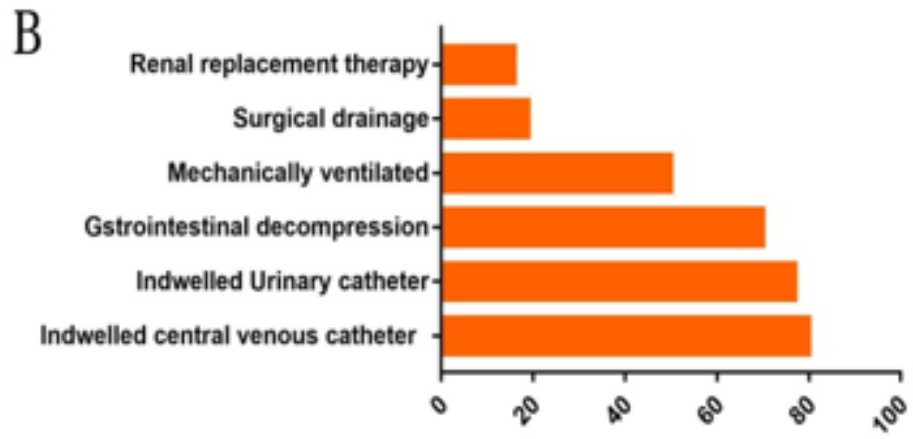

percentage of invasive Procedures

\section{Figure 2}

The percentage of CRKP BSIs patients from January 2016 to December 2018. A: percentage of CRKP BSIs patients' comorbidities; B: percentage of CRKP BSIs patients invasive Procedures

A

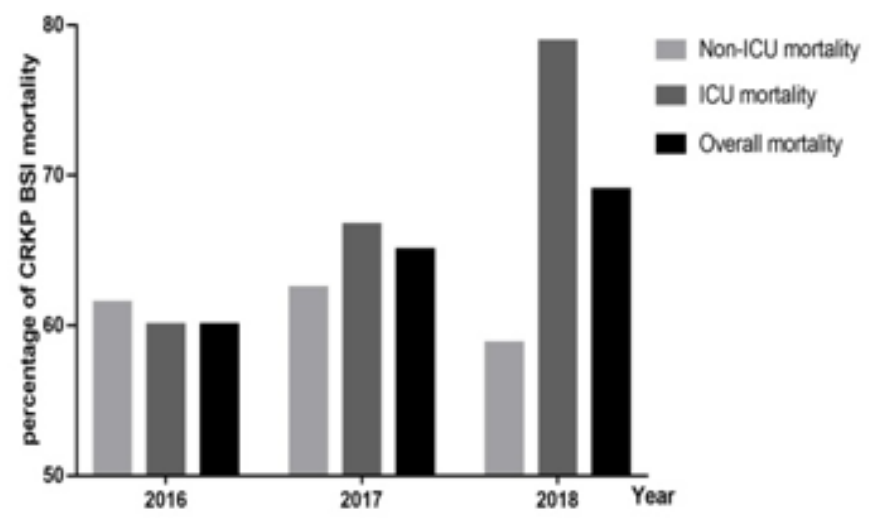

B

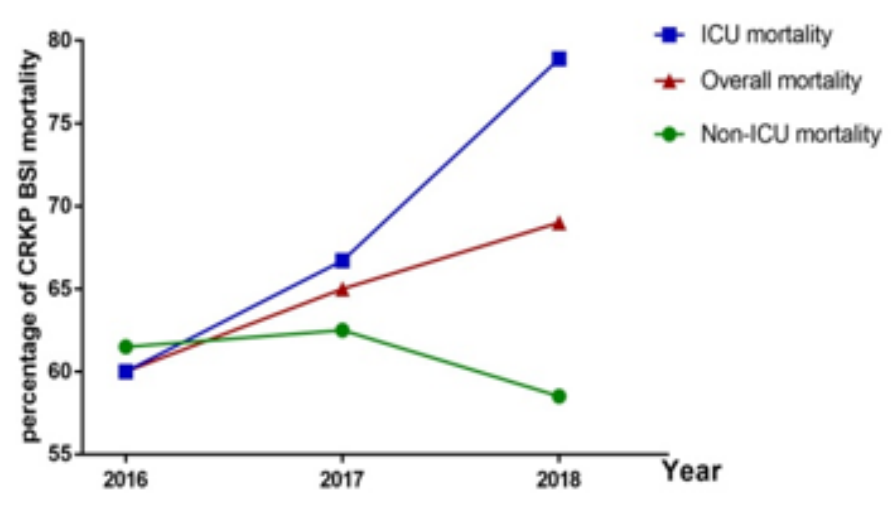

\section{Figure 3}

The percentage of CRKP BSIs mortality from January 2016 to December 2018. A: LightGray bar, percentage of CRKP-BSI mortality in our Hospital in Non-ICU; dark grey bar, percentage of CRKP-BSI mortality in our Hospital in intensive care unit; Black bar, percentage of CRKP-BSI overall mortality in our Hospital; B: Blue line, percentage of CRKP-BSI mortality in our Hospital in intensive care unit; Red line, percentage of CRKP-BSI overall mortality in our Hospital; Green line, percentage of CRKP-BSI mortality in our Hospital in Non-ICU. 


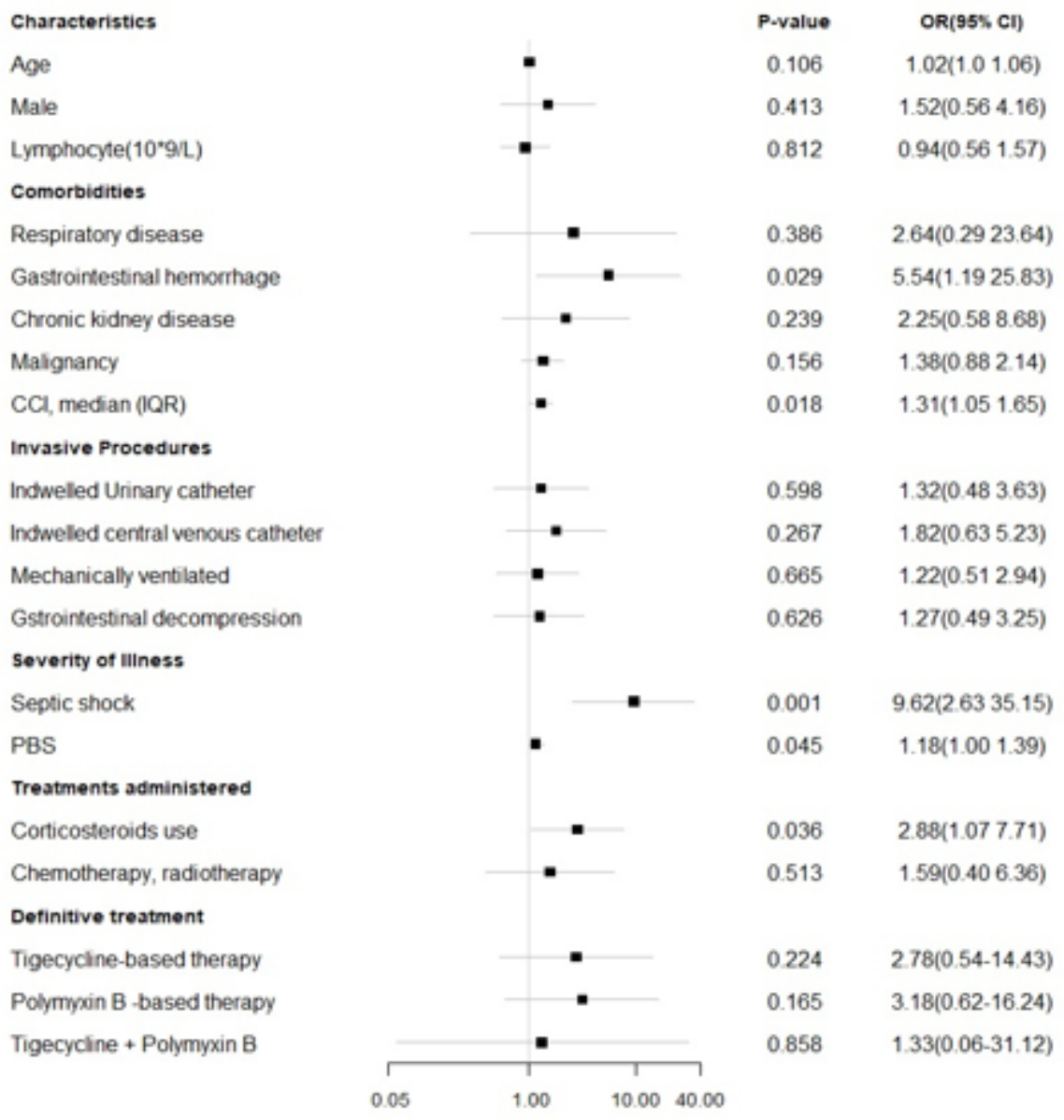

\section{Figure 4}

Crude odds ratio (OR) for the association mortality of patients with carbapenem-resistant Klebsiella pneumonia bloodstream infections 